\title{
ORTHOGONAL SERIES AND PROBABILITY
}

\section{DAN EUSTICE ${ }^{1}$}

1. Introduction. Recently R. Gundy [1] gave a characterization of Haar-like orthonormal systems based on martingale theory and used this theory to establish the capabilities of these systems for representing measurable functions. In this paper we will use this characterization of these orthonormal systems in deriving results related to recent work in Haar series [2], [3] and [4].

2. Definitions. Let $(X, \delta, \mu)$ be a probability space. [See [5] for probability theory concepts.] An orthonormal system $\left\{\phi_{n}\right\}$ on $(X, \delta, \mu)$ is called an $H$-system if the following conditions are satisfied:

(1) Each $\phi_{n}$ assumes at most two nonzero values with positive probability,

(2) the $\sigma$-field generated by $\left\{\phi_{n}\right\}_{n=1}^{N}$, written $\sigma\left(\phi_{1}, \phi_{2}, \cdots, \phi_{N}\right)$, consists of exactly $N$ atoms,

(3) $E\left(\phi_{n+1} \mid \phi_{1}, \cdots, \phi_{n}\right)=0, n \geqq 1$, that is, the conditional expectation of $\phi_{n+1}$ with respect to $\sigma\left(\phi_{1}, \cdots, \phi_{n}\right)$ is zero.

In [1] it is shown that this is equivalent to $\left\{\phi_{n}\right\}$ having the property, that for every $f$ in $L^{2}(X)$,

$$
E\left(f \mid \phi_{1}, \cdots, \phi_{n}\right)=\sum_{k=1}^{n} a_{k} \phi_{k}
$$

where $\left\{a_{k}\right\}$ are the Fourier coefficients of $f$ with respect to $\left\{\phi_{n}\right\}$.

$H$-systems are also easily seen to be equivalent to the class of orthonormal systems introduced by Price [3] and include the classical Haar system when the probability space is Lebesgue measure on $[0,1]$. [See [6] for the definition and some of the properties of the classical Haar system.]

3. Nonnegative Dirichlet kernels. If $\left\{\phi_{n}\right\}$ is an orthonormal system on $(X, \delta, \mu)$, then the Dirichlet kernels are

$$
D_{n}(x, y)=\sum_{i=1}^{n} \phi_{i}(x) \phi_{i}(y)
$$

Presented to the Society, January 24, 1967 under the title Haar series and probability; received by the editors April 14, 1966.

1 Research supported in part by the National Science Foundation. 
For the classical Haar system these kernels are nonnegative and this fact leads to the proof that the expansion of a continuous function on $[0,1]$ in a Haar series converges uniformly to the function. The following theorem, due to Price [3], has a more direct proof using probabalistic notions.

THEOREM 1. The Dirichlet kernels of an orthonormal system $\left\{\phi_{n}\right\}$ with $\phi_{1} \equiv 1$ are nonnegative if and only if $\left\{\phi_{n}\right\}$ is an H-system.

Proof. If $\left\{\phi_{n}\right\}$ is an $H$-system, then

$$
E\left(f \mid \phi_{1}, \cdots, \phi_{n}\right)=\sum_{k=1}^{n} a_{k} \phi_{k}=\int_{X} D_{n}(\cdot, t) f(t) d t
$$

for every nonnegative $f$ in $L^{2}(X, \S, \mu)$. Since the conditional expectation is a positive operator, the integral is nonnegative for every $f \geqq 0$ which implies $D_{n}(x, t) \geqq 0$.

To show that the Dirichlet kernels being nonnegative implies that $\left\{\phi_{n}\right\}$ is an $H$-system we will show by induction that the three conditions defining an $H$-system are satisfied..$^{2}$ For $n=1$ there is nothing to prove since $\phi_{1} \equiv 1$. Suppose the conditions are true for $2, \cdots, n$. Let $A_{1}, A_{2}, \cdots, A_{n}$ be the atoms of $\sigma\left(\phi_{1}, \cdots, \phi_{n}\right)$. Then, for fixed $s, D_{n}(s, t)$ must be constant for $t$ in each atom. Therefore, for $1 \leqq k \leqq n$ and $\chi_{A}$ denoting the characteristic function of $A$,

$$
\begin{aligned}
\chi_{A_{k}} & =E\left(\chi_{A_{k}} \mid \phi_{1}, \cdots, \phi_{n}\right)=\int_{X} \chi_{A_{k}}(t) D_{n}(\cdot, t) d \mu(t) \\
& =\left.\mu\left(A_{k}\right) D_{n}(\cdot, t)\right|_{\ell \in A_{k}} .
\end{aligned}
$$

Then,

$$
\begin{aligned}
D_{n}(s, t) & =1 / \mu\left(A_{k}\right),(s, t) \in A_{k} \times A_{k}, \quad k=1,2, \cdots, n, \\
& =0, \text { otherwise. }
\end{aligned}
$$

Since $D_{n+1}(s, t) \geqq 0, \phi_{n+1}(s) \phi_{n+1}(t) \geqq-D_{n}(s, t)$. Now, $\phi_{n+1}$ is zero on all but one of the atoms $A_{1}, A_{2}, \cdots, A_{n}$ since, if not, $\phi_{n+1}(s) \phi_{n+1}(t) \geqq 0$ everywhere by (1) and this contradicts the fact that $\int \phi_{n+1} d \mu=0$ because $\phi_{n+1}$ is orthogonal to $\phi_{1}$. Therefore, condition (3) of the definition is satisfied.

If $A_{k}$ is the atom of $\sigma\left(\phi_{1}, \cdots, \phi_{n}\right)$ on which $\phi_{n+1}$ is nonzero, set $P=\left\{x: \phi_{n+1}>0\right\}$ and $Q=A_{k}-P$. Let $c_{1}$ be the essential supremum of $\phi_{n+1}$ and $c_{2}$ be the essential infimum of $\phi_{n+1}$. The essential infimum of $\phi_{n+1}(s) \phi_{n+1}(t)$ for $s$ in $P$ and $t$ in $Q$ must be $c_{1} c_{2}$. Since $\phi_{n+1}$ is subject

${ }^{2}$ An alternate proof using idempotent contractions has been communicated to the author by R. F. Gundy. 
to the orthonormality conditions, the minimum value of $c_{1}$ occurs when $\phi_{n+1}(s) \equiv c_{1}$ a.e. on $P$ and the maximum value of $c_{2}$ occurs when $\phi_{n+1}(t) \equiv c_{2}$ a.e. on $Q$. In this case we find $c_{1} c_{2}=-1 / \mu\left(A_{k}\right)$. Therefore, if $\phi_{n+1}$ is not constant on $P$ and $Q$, the essential infimum of $\phi_{n+1}(s) \phi_{n+1}(t)$ is less than $-1 / \mu\left(A_{k}\right)$ which contradicts $D_{n+1}(s, t) \geqq 0$. Consequently, we have conditions (1) and (2) of the definition of an $H$-system also satisfied.

4. Convergence of $H$-series. It follows from the martingale convergence theorem $[5$, p. 331] that the expansion of an integrable function in an $H$-series converges a.e., converging to the function if the $H$-system is complete. Clearly, any sequence $\left\{a_{k}\right\}$ such that $\sum a_{k}^{2}<\infty$ is a coefficient sequence for which $\sum a_{k} \phi_{k}$ converges a.e. for any $H$-system $\left\{\phi_{n}\right\}$. We wish to examine $H$-series $\sum a_{k} \phi_{k}$ for which $\sum a_{\boldsymbol{k}}^{2}=\infty$.

An increasing sequence of atomic $\sigma$-fields is regular if there exists a $\delta>0$ such that for atoms $E_{n} \in \sigma(n)$ and $E_{n+1} \in \sigma(n+1)$ with $E_{n+1} \subset E_{n}$, $\mu\left(E_{n+1}\right) / \mu\left(E_{n}\right)>\delta$.

The following will prove useful.

LeMma 1 (Gundy). Let $\left\{\phi_{k}\right\}$ be an H-system such that $\sigma(n)$ $=\sigma\left(\phi_{1}, \cdots, \phi_{n}\right)$ is a regular sequence of $\sigma$-fields. Then the series $\sum a_{k} \phi_{k}$ converges a.e. on a set $E$ if and only if $\sum\left(a_{k} \phi_{k}\right)^{2}$ converges a.e. on $E$.

The proof (see Theorem 3.1 of [1]) uses several ideas from martingale theory.

Given $x$, let $E_{n}$ be the atom of $\sigma(n)$ containing $x$. If $\phi_{n}(x)=0$ for all $n$ sufficiently large then $\sum a_{k} \phi_{k}(x)$ converges for all sequences $\left\{a_{k}\right\}$. Therefore, let $E$ be the set of all $x$ for which the sequence of positive integers, $n_{k}=n_{k}(x)$, such that $\phi_{n_{k}}(x) \neq 0$ is infinite, and let $\epsilon_{1}=1$ and $\epsilon_{k}=1 / \mu\left(E_{n k-1}\right)$ for $k \geqq 2$.

ThEOREM 2. Let $\left\{\phi_{k}\right\}$ be an H-system satisfying the conditions of Lemma 1. Then $\sum a_{k} \phi_{k}$ converges a.e. on $E$ if and only if $\sum a_{n_{k}}^{2} \epsilon_{k}$ converges for almost all $x$ in $E$.

Proof. By the properties of $H$-systems, $\phi_{n_{k}}$ is nonzero only on $E_{n_{k} \Delta_{1}}$ and must be constant on $E_{n_{k}}$ and $E_{n_{k} \Delta_{1}}-E_{n_{k}}$. By the orthonormality of $\phi_{n}$, we have that on $E_{n_{k}}$,

$$
\phi_{n_{k}}^{2}=\mu\left(E_{n_{k-1}}-E_{n_{k}}\right) / \mu\left(E_{n_{k-1}}\right) \mu\left(E_{n_{k}}\right) .
$$

Since $\sigma(n)$ is regular, there exists a $\delta, 0<\delta<1$, such that

$$
\delta /(1-\delta) \mu\left(E_{n_{k-1}}\right) \leqq \phi_{n_{k}}^{2}(x) \leqq(1-\delta) / \delta \mu\left(E_{n_{k-1}}\right) .
$$


The theorem then follows by Lemma 1 .

For the remainder of this paper we will want to compare the behavior of $H$-systems with the classical Haar system and will assume that the measure space is the unit interval.

The following result, proved recently by Leindler [4], follows easily from Theorem 2.

COROLlaRY. If $\sum a_{\mathbf{k}}^{2}=\infty$ and $a_{\mathbf{k}+1}^{2} \leqq a_{\mathbf{k}}^{2}$, then $\sum a_{k} h_{k}$ diverges a.e., where $\left\{h_{k}\right\}$ is the Haar orthonormal system.

Proof. If $x$ is not a binary fraction, then

$$
\sum a_{n_{k}}^{2} \epsilon_{k}=\sum a_{n_{k}}^{2} 2^{k}
$$

where $2^{k}<n_{k} \leqq 2^{k+1}$. But this series diverges by Cauchy's condensation principle.

The preceding corollary does not hold for all $H$-systems, even if $\sigma(n)$ is a regular sequence of $\sigma$-fields.

THEOREM 3. Let $\mu$ be a nonatomic measure on $[0,1]$.

(a) If $\left\{a_{k}\right\}$ is a sequence such that $\lim \inf \left|a_{k}\right|=0$, then there exists an $H$-system, with $\sigma\left(\phi_{1}, \phi_{2}, \cdots\right)$ nonatomic, such that $\sum a_{k} \phi_{k}$ converges a.e.

(b) If $\left\{a_{k}\right\}$ is a sequence such that $\lim \sup \left|a_{k}\right|>0$, then there exists an $H$-system, with $\sigma\left(\phi_{1}, \phi_{2}, \cdots\right)$ nonatomic, such that $\sum a_{k} \phi_{k} d i$ verges a.e.

Proof. Let $I(0,0)=[0,1]$ and let $I(k, n), n \geqq 1,0 \leqq k \leqq 2^{n}-1$, denote a partition of $I(0,0)$ into disjoint sets of measure $2^{-n}$ such that $I(k, n-1)=I(2 k, n) \cup I(2 k+1, n)$. Choose a sequence of positive integers $\left\{p_{n}\right\}$ with the following properties:

$$
\begin{aligned}
p_{1} & \geqq 3, \\
\left|a_{p_{n}}\right| & <2^{-n}, \\
p_{n_{n}-n} & >q_{n},
\end{aligned}
$$

where $\left\{q_{n}\right\}$ denotes the sequence of positive in tegers complementary to $\left\{p_{n}\right\}$. We can assume that $\left\{q_{n}\right\}$ is infinite since otherwise $\sum a_{n}^{2}<\infty$ and $\sum a_{n} \phi_{n}$ converges a.e. for every $H$-system.

Let $\phi_{1}=1$ and assume $\left\{\phi_{k}\right\}$ has been defined for $k \leqq m-1$ in such a way that $\sigma\left(\phi_{1}, \cdots, \phi_{m-1}\right)$ is composed of atoms $I\left(k_{1}, n_{1}\right), I\left(k_{2}, n_{2}\right)$, $\cdots, I\left(k_{m-2}, n_{m-2}\right), I\left(2^{q}-1, q\right)$, where $n_{1}, n_{2}, \cdots, n_{m-2}, q$ are dependent on $m$ with $n_{1} \leqq n_{2} \leqq \cdots \leqq n_{m-2}$ and $I\left(2^{q}-1, q\right)$ is the unique atom that can be so expressed. If $m=p_{n}$ for some $n$, define 


$$
\begin{aligned}
\phi_{m} & =2^{n_{1} / 2} & & \text { on } I\left(2 k_{1}, n_{1}+1\right), \\
& =-2^{n_{1} / 2} & & \text { on } I\left(2 k_{1}+1, n_{1}+1\right), \\
& =0 & & \text { otherwise. }
\end{aligned}
$$

If $m \neq p_{n}$ for any $n$, then define $\phi_{m}$ similarly on $I\left(2^{q}-1, q\right)$. It is easily seen that $\left\{\phi_{m}\right\}$ is an $H$-system.

The sequence of atoms of the form $I\left(2^{q}-1, q\right)$ used in the construction form a decreasing sequence of sets and, since $\left\{q_{n}\right\}$ is infinite, their intersection, call it $Q$, has measure zero. Given $x, x$ not in $Q$, choose $M$ so large that the atom of $\sigma\left(\phi_{1}, \cdots, \phi_{M}\right)$ of the form $I\left(2^{q}-1, q\right)$ does not contain $x$. It can be seen from the definition of $\left\{\phi_{m}\right\}$, for $2^{j}-j \leqq n<2^{j+1}-(j+1)$ and $m=p_{n}$, that $\mu\left(\operatorname{support} \phi_{m}\right)=2^{-j}$. Therefore, using the notation of Theorem 2 ,

$$
\sum_{n_{k}>M} a_{n_{k}}^{2} \epsilon_{k} \leqq \sum_{p_{n}>M} a_{p_{n}}^{2} 2^{j} \leqq \sum\left(\frac{1}{2^{j}}\right)^{2} 2^{j}<\infty .
$$

By Theorem 2, $\sum a_{k} \phi_{k}$ converges a.e.

The proof of part (b) follows by an identical construction except that $\left\{a_{p_{n}}\right\}$ is chosen as a subsequence of $\left\{a_{n}\right\}$ which is bounded away from zero.

It is known [7] that there exist rearrangements of the Haar system such that the corresponding expansion of some function in $L^{2}(0,1)$ diverges a.e. As corollaries to the above constructions in Theorem 3, we can compare this to rearrangements of the Haar system which preserve the convergence a.e. of expansions of integrable functions.

COROLlaRy. If $\left\{a_{k}\right\}$ is a sequence such that $\lim \inf \left|a_{k}\right|=0$, then there exists a rearrangement of the Haar system, $\left\{\phi_{n}\right\}$, which is an $H$-system, for which $\sum a_{k} \phi_{k}$ converges a.e.

Corollary. If $\left\{a_{k}\right\}$ is a sequence such that $\lim \sup \left|a_{k}\right|>0$, then there exists a rearrangement of the Haar system, $\left\{\phi_{n}\right\}$, which is an $H$ system, for which $\sum a_{k} \phi_{k}$ diverges a.e.

Proof. If the sets $I(k, n)$ are chosen to be the intervals $\left(k / 2^{n},(k+1) / 2^{n}\right)$, then the orthonormal system constructed in Theorem 3 is a rearrangement of the Haar system and the corollaries then follow.

5. Sets of completeness for $H$-systems. In this section we will assume that $\mu$ is Lebesgue measure on $[0,1]$.

An $H$-system need not be complete on $L^{2}(0,1)$. For example, the 
$\sigma$-field generated by $\left\{\phi_{n}\right\}$, denoted by $\sigma\left(\phi_{1}, \phi_{2}, \cdots\right)$, could contain atoms. In [2], Price and Zink characterized the subsets of [0,1] on which a subsystem of the classical Haar system is complete.

Let $G$ be a measurable subset of $[0,1]$. A system of functions $\{\mathfrak{F}\}$ is total in measure on $G$ if for every measurable function $f$ on $G$ there is a sequence of finite linear combinations of functions of $\{\mathfrak{F}\}$ that converges in measure to $f$.

The following theorems of Talalyan compare totality in measure with completeness.

Theorem A. A system of functions $\{\mathfrak{F}\}$ is total in measure on a measurable subset $G$ of $[0,1]$ if and only if to each $\epsilon>0$, there corresponds a measurable subset $E$ of $G$ such that $\mu(E) \geqq \mu(G)-\epsilon$ and $\{\mathfrak{F}\}$ is complete in $L^{2}(E)$.

THEOREM B. If a sequence of measurable functions is total in measure on a measurable subset of $[0,1]$, then it remains so when a finite set of functions is removed from it.

For the proofs, see [8] and [9].

Given a sequence of measurable functions $\left\{f_{n}\right\}$, let the lim sup (support $f_{n}$ ) be called the support of $\left\{f_{n}\right\}$. Two measurable sets will be called equivalent if they differ by sets of measure zero.

Theorem 4. A subsystem $\left\{\phi_{n}\right\}$ of an $H$-system is total in measure on a measurable set $G$ in $[0,1]$ if and only if $G$ is equivalent to a set in $\sigma\left(\phi_{1}, \phi_{2}, \cdots\right)$ which is contained in the support of $\left\{\phi_{n}\right\}$.

Proof. If $\left\{\phi_{n}\right\}$ is total in measure on $G$ then $\chi_{G}$ is the limit in measure of a sequence of functions, $\left\{f_{n}\right\}$, where each $f_{n}$ is measurable with respect to $\sigma\left(\phi_{1}, \cdots, \phi_{n}\right)$ and hence $G$ is equivalent to a set in $\sigma\left(\phi_{1}, \phi_{2}, \cdots\right)$. By Theorem B, $\chi_{G}$ equals a.e. a function measurable with respect to $\sigma\left(\phi_{n+1}, \phi_{n+2}, \cdots\right)$. This implies almost all $x$ in $G$ are in the support of at least one $\phi_{k}, k \geqq n+1$ and, hence, in the lim sup (support $\phi_{n}$ ).

Conversely, if $G$ is in $\sigma\left(\phi_{1}, \phi_{2}, \cdots\right)$ and in the support of $\left\{\phi_{n}\right\}$, then $f_{n}=E\left(\chi_{G} \mid \phi_{1}, \cdots, \phi_{n}\right)$ converges to $\chi_{a}$ a.e. by the martingale convergence theorem $\left[5\right.$, p. 331]. Since each $\sigma\left(\phi_{1}, \cdots, \phi_{n}\right)$ is composed of a finite collection of atoms, each $f_{n}$ is a finite linear combination of $\phi_{1}, \cdots, \phi_{n}$ and so $\left\{\phi_{n}\right\}$ is total in measure on $G$.

REMARK. Theorem 4 will hold for every measurable subset of the support of $\left\{\phi_{n}\right\}$ if $\sigma\left(\phi_{1}, \phi_{2}, \cdots\right)$ restricted to the support of $\left\{\phi_{n}\right\}$ is equivalent to the Borel $\sigma$-algebra. That this happens if $\left\{\phi_{n}\right\}$ is a subsystem of the Haar functions is a result of Price and Zink [2] and we obtain it as a corollary. 
CoROllaRy. Let $\left\{\phi_{n}\right\}$ be a subsystem of the Haar functions. $\left\{\phi_{n}\right\}$ is total in measure on a set $G$ if and only if almost all points of $G$ are contained in the support of $\left\{\phi_{n}\right\}$.

Proof. Let $E$ denote the support of $\left\{\phi_{n}\right\}$ and let

$$
\psi_{n}=E\left(\chi_{G} \mid \phi_{1}, \cdots, \phi_{n}\right) .
$$

Since the support of each $\phi_{n}$ is an interval, the atoms of $\sigma\left(\phi_{1}, \cdots, \phi_{n}\right)$ are intervals. Given a point $x$, let $I_{n}(x)$ denote the interval of $\sigma\left(\phi_{1}, \cdots, \phi_{n}\right)$ containing $x$. For points in $E, \lim _{n \rightarrow \infty} \mu\left(I_{n}(x)\right)=0$. From the definition of conditional expectation, $\psi_{n}(x)=\mu\left(G \cap I_{n}(x)\right) / \mu\left(I_{n}(x)\right)$, and the martingale convergence theorem implies

$$
\lim _{n \rightarrow \infty} \psi_{n}=E\left(\chi_{G} \mid \phi_{1}, \phi_{2}, \cdots\right) \text { a.e. }
$$

To see that $\lim _{n \rightarrow \infty} \psi_{n}=\chi_{G}$ a.e., we observe that on the set where $\lim _{n \rightarrow \infty} \mu\left(I_{n}(x)\right)=0, \psi_{n}(x)$ converges, except for a set of measure zero, to 1 for $x$ in $G$ and to 0 for $x$ not in $G$ by the density theorem; while on the set where $\lim _{n \rightarrow \infty} \mu\left(I_{n}(x)\right)=\alpha$, for some $\alpha>0$, $\lim _{n \rightarrow \infty} \mu\left(I_{n}(x) \cap G\right)=0$ since $\lim _{n \rightarrow \infty} I_{n}(x) \cap G=\varnothing$. Therefore, $G$ is equivalent to a set in $\sigma\left(\phi_{1}, \phi_{2}, \cdots\right)$.

\section{REFERENCES}

1. R. F. Gundy, Martingale theory and pointwise convergence of certain orthogonal series, Trans. Amer. Math. Soc. 124 (1966), 228-248.

2. J. J. Price and R. E. Zink, On sets of completeness for families of Haar functions, Trans. Amer. Math. Soc. 119 (1965), 262-269.

3. J. J. Price, Orthonormal sets with non-negative Dirichlet kernels, Trans. Amer. Math. Soc. 95 (1960), 256-262.

4. L. Leindler, Über Konvergenz- und Summationeigenschaften von Haaren Reihen, Acta. Math. Sci. (Szeged) 26 (1965), 19-30.

5. J. L. Doob, Stochastic processes, Wiley, New York, 1953.

6. G. Alexits, Convergence problems of orthogonal series, Pergamon Press, New York, 1961.

7. P. L. Ulyanov, Divergent fourier series, Uspehi Mat. Nauk. 16 (1961), 61-142.

8. A. A. Talalyan, On the convergence a.e. of subsequences of partial sums of general orthogonal series, Izv. Akad. Nauk Armjan. SSR Ser. Fiz.-Mat. Nauk 10 (1957), 17-34.

9. C. Goffman and D. Waterman, Basic sequences in the space of measurable functions, Proc. Amer. Math. Soc. 11 (1960), 211-213.

UNIVERSITY OF ILLINOIS AND

The Ohio State University 